\title{
Suplemento artesanal oral: uma proposta para recuperação nutricional de crianças e adolescentes com câncer ${ }^{1}$
}

\author{
Homemade oral supplement: a proposal \\ for the nutritional recovery of children \\ and adolescents with cancer
}

Fernanda Rodrigues ALVES²

Adriana GARÓFOLO ${ }^{3}$

Priscila dos Santos MAIA ${ }^{4}$

Fernando José de NÓBREGA ${ }^{5}$

Antonio Sergio PETRILLI4

RE S U M O

\section{Objetivo}

Avaliar o impacto do suplemento oral artesanal na recuperação do estado nutricional de pacientes com desnutrição leve, grave e com risco nutricional.

\section{Métodos}

Propuseram-se oito receitas de suplementos visando ofertar entre 30,0\% e 35,0\% do gasto energético total. Os pacientes com desnutrição grave (grupo B) receberam o suplemento oral por duas semanas, e os demais pacientes (grupo A), por quatro semanas. Para a comparação dos resultados obtidos com o emprego do suplemento oral artesanal, foram utilizados dados referentes a um protocolo anterior, com o mesmo desenho, entretanto, com a utilização de suplemento oral industrializado.

\section{Resultados}

O suplemento oral artesanal fica muito aquém no que diz respeito a alguns micronutrientes, entretanto é cinco vezes mais barato do que a preparação com o suplemento oral industrializado. Os pacientes do grupo A com suplemento oral artesanal apresentaram $88,0 \%$ de resposta positiva na semana de avaliação, enquanto os

\footnotetext{
1 Artigo elaborado a partir da dissertação de F.R. ALVES, intitulada "Suplemento artesanal oral: uma proposta para recuperação nutricional de crianças e adolescentes com câncer". Universidade Federal de São Paulo; 2008.

${ }^{2}$ Hospital Samaritano de São Paulo. R. Conselheiro Brotero, 1486, Higienópolis, 01232-010, São Paulo, SP, Brasil.

Correspondência para/Correspondence to: F.R. ALVES. E-mail: <nanda.nutri@gmail.com>.

3 Instituto Adriana Garófolo. São Paulo, SP, Brasil.

${ }^{4}$ Universidade Federal de São Paulo, Departamento de Pediatria, Instituto de Oncologia Pediátrica. São Paulo, SP, Brasil.

${ }^{5}$ Universidade Federal de São Paulo, Departamento de Pediatria. São Paulo, SP, Brasil.
} 
com suplemento oral industrializado tiveram $84,0 \%$. No grupo $B$, foram recuperados $22,0 \%$ dos pacientes com suplemento oral artesanal e $25,0 \%$ do grupo com suplemento oral industrializado, não apresentando, portanto, diferença significante. Comparando o impacto do industrializado com o do artesanal na prega cutânea tricipital e circunferência do braço, verificou-se que o suplemento oral industrializado no grupo A apresentou melhores resultados que o suplemento oral artesanal, e no grupo B, esse efeito observado na prega cutânea não foi significante $(p=0,16)$. Os consumos de energia e de proteína, assim como a evolução nutricional, foram semelhantes entre suplemento oral industrializado e suplemento oral artesanal. Apenas a composição corpórea no grupo A com suplemento oral industrializado apresentou melhores resultados.

\section{Conclusão}

Os resultados apresentados neste estudo sugerem que o emprego da terapia com suplemento artesanal seja uma opção capaz de auxiliar na recuperação nutricional de pacientes oncológicos e uma opção para populações financeiramente desfavorecidas.

Termos de indexação: Adolescentes. Neoplasias. Criança. Desnutrição. Suporte nutricional.

\section{A B S T R A C T}

\section{Objective}

The aim of this study was to evaluate the impact of homemade oral supplements on the nutritional recovery of patients with mild or severe malnutrition or at nutritional risk.

\section{Methods}

Eight recipes of homemade oral supplements containing 30\% to 35\% of the total energy expenditure were proposed. The patients with severe malnutrition (group B) received the oral supplement for 2 weeks and the others for 4 weeks (group A). Oral supplementation with homemade supplements was compared with oral supplementation with store-bought supplements, investigated earlier with a protocol with the same design.

\section{Results}

Homemade oral supplements contain much lower amounts of certain micronutrients but are five times cheaper than store-bought supplements. In group A, $88 \%$ of the patients taking homemade oral supplements and $84 \%$ of the patients taking store-bought supplements responded positively to supplementation. In group $B$, $22 \%$ of the patients taking homemade oral supplements and $25 \%$ of the patients taking store-bought supplements recovered. The difference was not significant. The impact of store-bought supplementation on the triceps skinfold thicknesses and arm circumferences of the patients in group A was greater than that obtained with homemade supplements. In group $B$, the effect on triceps skinfold thickness was not significant $(p=0.16)$. Patients taking homemade or store-bought oral supplements presented similar protein and energy intakes and improvements in nutritional status. Only the body composition of patients in group A taking store-bought oral supplements was better.

\section{Conclusion}

The results obtained by this study suggest that the therapeutic use of homemade oral supplements is an alternative capable of promoting the nutritional recovery of cancer patients, especially those who cannot afford store-bought supplements.

Indexing terms: Adolescents. Neoplasms. Child. Malnutrition. Nutritional support.

\section{N T R O D U Ç Ã O}

O câncer da criança e do adolescente compreende 3,0\% de todas as neoplasias malignas humanas ${ }^{1}$. Estima-se que o câncer em pacientes com idade inferior a 20 anos seja de 16 casos por 100 mil habitantes². As neoplasias na infância mais incidentes são as leucemias, segui- das dos tumores do sistema nervoso central e linfomas'.

A desnutrição é a principal complicação nutricional nos pacientes com câncer e tem sido apontada por vários autores ${ }^{3-7}$. O grau de comprometimento nutricional está associado com o tipo de tumor, com o estágio da doença e com os 
órgãos acometidos ${ }^{8}$. Os estudos indicam grande variabilidade na prevalência da desnutrição, chegando até 50,0\% em pacientes pediátricos oncológicos ${ }^{3-5,7}$.

A quimioterapia e a radioterapia provocam alterações gastrintestinais importantes, tais como náusea, vômito, mucosite, diarreia, alteração no paladar, xerostomia, e alteração na absorção e metabolização dos nutrientes. Isso pode levar à redução da ingestão alimentar, além de instalação de aversões a alimentos específicos ${ }^{8-12}$. Os pacientes desnutridos apresentam maiores taxas de morbi-mortalidade, independentemente da base associada, especialmente quando o grupo desnutrido é composto por crianças, 13-17. Sawaya apresentou dados de prevalência de infecção: 90,0\% dos pacientes com desnutrição grave tiveram pelo menos um episódio infeccioso no período de um mês ${ }^{18}$. Além de favorecer o prognóstico, a melhora do estado nutricional também parece estar associada com a melhora da qualidade de vida. Os dados da literatura sugerem que o estado nutricional adequado está associado com maior sobrevida, menos tempo de hospitalização e maior tolerância ao tratamento oncológico proposto $5,18,19$.

A introdução de suplementos orais pode melhorar a ingestão dietética, que geralmente está comprometida e conferindo menor oferta de energia e nutrientes. Os suplementos ofertam energia, proteína e outros nutrientes, sendo um bom meio para suprir as necessidades nutricionais comprometidas 9,12. Em um estudo-piloto com pacientes adultos com câncer, foi verificado que após oito semanas de intervenção nutricional, com suplemento industrializado por via oral, $80 \%$ dos pacientes desnutridos foram recuperados ${ }^{18}$. A terapia nutricional com suplemento industrializado apresenta alto custo e, portanto, não é uma realidade sustentável por populações menos favorecidas e, até mesmo, por alguns centros de tratamento. Os indicadores sociais do Brasil demonstram alta prevalência de pobreza ${ }^{20}$. Os suplementos artesanais são obtidos a partir da modulação de ingredientes dietéticos e constituem-se uma opção menos onerosa e de aplicação mais ampla para populações de menor renda. Por isso, o objetivo deste estudo foi avaliar o impacto do Suplemento Oral Artesanal (SOA) na recuperação do estado nutricional de pacientes com desnutrição leve, grave e com risco nutricional. Para tal objetivo serão verificadas as seguintes variáveis: adesão ao protocolo de tratamento, frequência de internação hospitalar, presença de toxicidade gastrintestinal, bem como ingestão alimentar, consumo do suplemento, evolução do escore de capacidade funcional e evolução das pregas e circunferências. Esses resultados foram comparados com os dados de um protocolo anterior que utilizou Suplemento Oral Industrializado (SOI).

\section{M É T O D O S}

O estudo foi desenvolvido com crianças e adolescentes com comprometimento nutricional e com câncer em tratamento no Instituto de Oncologia Pediátrica do Departamento de Pediatria da Escola Paulista de Medicina, Universidade Federal de São Paulo. O acompanhamento foi realizado no período de maio de 2006 a outubro de 2007, quando foi feita a coleta de dados do SOA. Os dados do SOI foram coletados anteriormente, nos anos de 2004 e 2005. Foram incluídos pacientes com neoplasia maligna em tratamento oncológico e com déficit nutricional caracterizado pelos seguintes cortes: a) desnutrição leve (DEP leve): Índice de Massa Corporal (IMC) menor que o percentil quinze e maior que o cinco para adolescentes. A classificação de desnutrição leve para crianças define-se pelo escore- $Z$ de Peso para Estatura (P/E) $<-1,0$ e $\geq-2,0$ desvios-padrão; b) risco nutricional: pacientes eutróficos com perda de peso igual ou superior a $5 \%$ em um período de até seis meses; c) desnutrição grave (DEP grave): IMC abaixo do quinto percentil para adolescentes e escore-Z de P/E $<-2,0$ desvios-padrão para crianças.

Em pacientes com peso superestimado por massa tumoral abdominal, o estado nutricional foi diagnosticado por meio da Prega Cutânea 
Tricipital (PCT) e Circunferência do Braço (CB). Os pacientes foram considerados desnutridos graves quando apresentaram esses indicadores abaixo do quinto percentil. Na metodologia, o indicador de Circunferência Muscular do Braço (CMB) não foi utilizado como valor de corte, pois o CMB é uma estimativa obtida por meio de cálculo realizado com os valores de PCT e CB, sendo utilizados apenas esses indicadores para a análise de dados antropométricos.

Os pacientes foram divididos em dois grupos: o grupo A contemplou os pacientes com diagnóstico nutricional de desnutrição leve e os considerados de risco nutricional, e o grupo B incluiu os pacientes com diagnóstico de desnutrição grave e aqueles com peso superestimado por massa tumoral e com PCT e CB abaixo do quinto percentil.

Critérios de exclusão: pacientes que, por condições clínicas, não podiam receber algum ingrediente da receita ou das orientações dietéticas padronizadas; pacientes em uso de corticosteróides; limitações físicas para ingestão alimentar adequada.

Tratou-se de um ensaio clínico prospectivo não controlado, no qual os pacientes eram incluídos em série durante todo o período do protocolo. Os pacientes foram acompanhados semanalmente no ambulatório de nutrição e nas unidades de internação quando eram eventualmente internados. A resposta nutricional (desfecho) foi verificada na semana dois para os pacientes do grupo $B$ e na quatro para os do grupo $A$. O desfecho positivo foi verificado por meio dos resultados favoráveis à terapia nutricional oral, não havendo necessidade de indicação de sonda. Os pacientes do grupo A deveriam estar eutróficos ou com desnutrição leve e não deveriam evoluir para DEP grave. Para os pacientes do grupo B, o objetivo era o aumento do escore- $Z$ em $\geq 0,3$ desvios-padrão do peso para a estatura em crianças e aumento de $\geq 3,0 \%$ de adequação do IMC para os adolescentes. No caso dos pacientes com peso superestimado por massa tumoral, o critério para considerar desfecho positivo foi o aumento nos valores de PCT e CB acima do quinto percentil.
A orientação dietética foi individualizada e também realizou-se orientação de suplementação lipídica - adição de uma colher de sopa de óleo, azeite, margarina ou maionese sobre a comida no almoço e jantar do paciente - e proteica - com o consumo de um a dois ovos por dia. Para o suplemento oral artesanal foram desenvolvidas oito receitas com base em quatro ingredientes alimentares (leite, ovo, açúcar e óleo); a finalidade foi aumentar a oferta de energia e melhorar a oferta proteica. A orientação do consumo do suplemento visou oferecer entre 30 e $35 \%$ do gasto energético total. Depois de calculadas para verificação da composição nutricional, as receitas foram previamente testadas para verificação da palatabilidade. O valor nutricional de proteína e de energia foi comparado às recomendações para pacientes pediátricos com câncer ${ }^{21,22}$, para os micronutrientes, foram utilizadas as Dietary Recommended Intakes (DRI) ${ }^{23,24}$. Para a avaliação da composição nutricional dos suplementos artesanais foram estimados os volumes de suplemento ingeridos por faixa etária e sexo.

Para a comparação dos resultados obtidos com o emprego do suplemento artesanal, foram utilizados dados referentes a um protocolo anterior, desenvolvido prospectivamente e avaliado de forma histórica neste estudo. O desenho desse protocolo foi o mesmo, mas utilizou-se suplemento industrializado no lugar do artesanal. Os suplementos utilizados foram o nutren 1.0 (para adolescentes) e nutren jr. (para crianças), ambos na forma de pó, diluídos em leite e com acréscimo de outros ingredientes para dar sabor, como fruta ou achocolatado. Esses suplementos foram doados aos pacientes.

As toxicidades gastrintestinais foram avaliadas semanalmente, sendo verificada a frequência ocorrida de náusea/vômito, diarréia e mucosite.

Foi avaliada a capacidade funcional por meio das escalas de Lansky et al. ${ }^{25}$ para pacientes com idade inferior ou igual a dezesseis anos, e de Karnofsky \& Burchenal ${ }^{26}$ para pacientes com idade superior a dezesseis anos. Para todo o grupo foi 
aplicada a escala de European Cooperative Oncology Group (ECOG) ${ }^{27}$.

A situação social foi verificada com o objetivo de demonstrar a necessidade de uma proposta menos onerosa para as famílias. Para isso, coletamos o grau de instrução dos cuidadores (pais ou responsáveis) em ambos os protocolos.

A ingestão alimentar foi coletada por meio de recordatório alimentar realizado semanalmente, no qual a nutricionista verificava a ingestão alimentar habitual ${ }^{28}$. Os dados coletados foram calculados para avaliação da ingestão de energia e de proteína.

A dor foi mensurada semanalmente nos pacientes do protocolo de suplemento artesanal. A medida utilizada foi a escala numérica que varia entre 0 e 10 , sendo 10 referente à dor de maior intensidade que o paciente já sentiu ${ }^{29-31}$.

\section{Análise estatística}

Para avaliar a evolução entre a semana inicial e a do desfecho, foi aplicado o teste T para amostras pareadas quando os dados estiveram distribuídos em uma curva normal. Quando a normalidade foi rejeitada, foi utilizado o teste de postos de Wilcoxon. Para comparar a evolução entre os dois grupos - artesanal e industrializado -, aplicou-se o teste T para amostras não pareadas no caso de normalidade e Mann' Whitney caso a distribuição dos dados não fosse normal. Aplicou-se o teste de Qui-quadrado para dados categóricos a fim de verificar se os pacientes dos grupos SOI e SOA foram diferentes quanto à escolaridade dos cuidadores, diagnósticos, sexo e faixa etária. A análise de regressão múltipla foi aplicada para as análises de associação, sendo utilizadas como variáveis potenciais de confundimento de sexo, idade, escolaridade, diagnóstico e nível econômico. Para as análises de regressão, o número de variáveis não foi superior a $15 \%$ do número de indivíduos do estudo. Todos os testes foram bicaudais com o valor de $p<0,05$ considerado como significante ${ }^{32}$.

\section{Aspectos éticos}

O estudo recebeu aprovação do Comitê de Ética em Pesquisa da Escola Paulista de Medicina - Unifesp em 26 de maio de 2006 sob o número de protocolo $0163 / 06$. Todos os pacientes foram esclarecidos sobre os riscos e os benefícios das técnicas e intervenções e assinaram o termo de consentimento livre e esclarecido para autorização.

\section{RESULTADOS}

Foram admitidos 115 pacientes com média de idade de 12 anos, sendo 40,0\% crianças e $60,0 \%$ adolescentes. Sessenta e um por cento dos pacientes eram do sexo masculino e $39,0 \%$, do feminino. Foram excluídos cinco pacientes por necessidade de dieta via sonda na semana zero do protocolo. Com relação ao diagnóstico, 15,5\% (17/110) dos pacientes tinham doenças hematológicas malignas, e 84,5\% (93/110), tumores sólidos não hematológicos. Foram avaliados 110 pacientes, sendo 50,0\% (55/110) do grupo A e $50,0 \%$ (55/110) do grupo B (Tabela 1). Cinquenta pacientes receberam suplemento industrializado; desses, um foi excluído por necessidade de dieta via sonda na semana zero. Foram avaliados 49 pacientes: $51,0 \%$ (25/49) do grupo B e $49,0 \%$ (24/49) do grupo A, sendo a média de idade de 12 anos. Os grupos SOI e SOA não são diferentes quanto ao sexo, idade e diagnóstico dos pacientes.

Oitenta e um por cento dos cuidadores do grupo com SOI e $71,0 \%$ dos com SOA tinham grau de instrução entre analfabeto e ensino fundamental completo. Dos 110 pacientes do grupo SOA, cinco foram excluídos da análise do desfecho, sendo dois por óbito e três por abandono antes da semana de avaliação, totalizando um grupo de 105. No grupo que recebeu SOI, dos 49 pacientes admitidos, seis não completaram o protocolo, sendo avaliados 43 pacientes.

A quantidade de energia por mililitro de suplemento artesanal variou de 1,35 a 2,17kcal, 
ofertando em média $1,76 \mathrm{kcal} / \mathrm{mL}$. A distribuição de macronutrientes foi da ordem de $39,0 \%$ a $59,0 \%$ de carboidrato, $11,0 \%$ a $13,0 \%$ de proteína e 30,0\% a 49,0\% de lipídeo. A proteína e 11 dos 23 micronutrientes dos suplementos artesanais atingiram em média $70,0 \%$ ou mais das recomendações. A vitamina $C$, vitamina $K$, ácido fólico e manganês apresentaram média de adequação criticamente baixa, ou seja, inferior a $15,0 \%$ da recomendação. Entretanto, tiamina, riboflavina, vitamina $B_{12}$, vitamina $B_{6}$ e biotina apresentam médias percentuais de adequação acima da recomendação. A comparação com o suplemento industrializado mostrou que as vitaminas C e K, ácido fólico, biotina, cobre, manganês, magnésio e ferro estão em menor quantidade no suplemento artesanal, com menos de $25,0 \%$ dos valores encontrados no industrializado. O percentual de energia do suplemento industrializado - 54,0\% - ultrapassa o suplemento

Tabela 1. Características demográficas dos pacientes nos grupos SOI e SOA, separados por estado nutricional.

\begin{tabular}{|c|c|c|c|c|c|}
\hline \multirow{2}{*}{ Características } & & \multicolumn{2}{|c|}{ Grupo A } & \multicolumn{2}{|c|}{ Grupo B } \\
\hline & & $\mathrm{n}$ & Percentual & $\mathrm{n}$ & Percentual \\
\hline \multirow[t]{6}{*}{ Idade } & SOA & 55 & 100,0 & 55 & 100,0 \\
\hline & Crianças & 24 & 40,0 & 22 & 40,0 \\
\hline & Adolescentes & 31 & 60,0 & 37 & 60,0 \\
\hline & SOI & 24 & 100,0 & 25 & 100,0 \\
\hline & Crianças & 10 & 32,5 & 7 & 32,5 \\
\hline & Adolescentes & 14 & 67,5 & 18 & 67,5 \\
\hline \multirow[t]{18}{*}{ Sexo } & SOA & 55 & 100,0 & 55 & 100,0 \\
\hline & Masculino & 31 & 61,0 & 38 & 61,0 \\
\hline & Feminino & 24 & 39,0 & 21 & 39,0 \\
\hline & SOI & 24 & 100,0 & 25 & 100,0 \\
\hline & Masculino & 15 & 63,0 & 16 & 63,0 \\
\hline & Feminino & 9 & 37,0 & 9 & 37,0 \\
\hline & SOA & 55 & 100,0 & 55 & 100,0 \\
\hline & Leucemias & 5 & 9,0 & 3 & 5,5 \\
\hline & Linfomas & 2 & 3,5 & 7 & 12,5 \\
\hline & Tumores ósseos & 16 & 29,0 & 16 & 29,0 \\
\hline & Tumores do sistema nervoso central & 13 & 24,0 & 3 & 5,5 \\
\hline & Neuroblastoma & 6 & 11,0 & 7 & 13,0 \\
\hline & Tumores renais & 4 & 7,5 & 9 & 16,5 \\
\hline & Sarcomas de partes moles & 3 & 5,5 & 2 & 3,5 \\
\hline & Tumores de células germinativas & 2 & 3,5 & 5 & 9,0 \\
\hline & Retinoblastoma & 2 & 3,5 & 0 & 0 \\
\hline & Carcinoma & 0 & 0 & 1 & 2,0 \\
\hline & Outros & 2 & 3,5 & 2 & 3,5 \\
\hline \multirow[t]{12}{*}{ Diagnósticos } & SOI & 24 & 100,0 & 25 & 100,0 \\
\hline & Leucemias & 4 & 16,5 & 4 & 16,0 \\
\hline & Linfomas & 2 & 8,0 & 2 & 8,0 \\
\hline & Tumores ósseos & 5 & 21,0 & 7 & 28,0 \\
\hline & Tumores do sistema nervoso central & 5 & 21,0 & 1 & 4,0 \\
\hline & Neuroblastoma & 1 & 4,0 & 1 & 4,0 \\
\hline & Tumores renais & 3 & 12,5 & 1 & 4,0 \\
\hline & Sarcomas de partes moles & 2 & 8,5 & 3 & 12,0 \\
\hline & Tumores de células germinativas & 1 & 4,0 & 0 & 0 \\
\hline & Retinoblastoma & 0 & 0,0 & 0 & 0 \\
\hline & Carcinoma & 1 & 4,0 & 4 & 16,0 \\
\hline & Outros & 0 & 0 & 2 & 8,0 \\
\hline
\end{tabular}

*Não há diferenças estatisticamente significantes entre os grupos SOI e SOA (teste de Qui-quadrado com dados categorizados). SOA: suplementação oral artesanal; SOI: suplementação oral industrializada. 
artesanal - 43,0\% - em 11,0\%. Assim também ocorre com a oferta proteica total: $91,0 \%$ no industrializado e $77,0 \%$ no artesanal.

Em relação ao custo dos suplementos, o industrializado custa cinco vezes mais do que o artesanal. O valor de aproximadamente $500 \mathrm{~mL}$ consumidos diariamente por uma semana é de $R \$ 62,25$ para o industrializado e de $R \$ 13,11$ para o artesanal (dados referentes ao mês de agosto de 2007).

Os pacientes do grupo A com SOA apresentaram $88,0 \%$ de resposta positiva (desfecho) na semana de avaliação, enquanto os com SOI apresentaram $84,0 \%$. No grupo $B$ foram recuperados $22,0 \%$ dos pacientes com SOA para $25,0 \%$ do grupo com SOI. Ambas as avaliações não apresentaram diferença significante (Figura 1). A análise de associação (regressão múltipla) entre a ingestão do suplemento industrializado e a evolução do estado nutricional verificou associação positiva: quanto maior foi o consumo do suplemento, melhor foi a evolução nutricional $(r=1,24$; IC $-6,36$ a $-0,54$ e $p=0,008)$.

Avaliando-se a adequação da PCT, CB e $C M B$, observou-se melhora na evolução da $C B$ e $C M B$ do grupo $A$, tanto para os que receberam SOI quanto para os que receberam SOA, entretanto com impacto menos importante do SOA e valores estatisticamente significantes apenas nos pacientes com SOI (CB: $p=0,014$ e CMB: $p=0,0041)$. A comparação da evolução da PCT do grupo SOI e do SOA não demonstrou diferença. A evolução da $C B$ e CMB dos pacientes do grupo $B$ não apresentou diferença, entretanto a evolução da PCT foi melhor nos pacientes desse grupo que receberam SOI $(p=0,04)$ (Tabela 2$)$.

Comparando o impacto do SOI e do SOA nesses indicadores, verificou-se que o SOI no grupo $\mathrm{A}$ apresentou melhores resultados que $\mathrm{O}$ SOA, sendo estatisticamente significante (PCT: $p=0,024, C B: p=0,011$ e CMB: $p=0,01)$. No grupo $B$, esse efeito foi observado na PCT, porém sem significância estatística $(p=0,16)$. Dentre as análises de associação realizadas com esses indicadores, verificamos associação positiva entre $C B$ e ingestão do suplemento industrializado (grupo A e B) $(r=3,14$; IC 7,97 a 6,19 e $p=0,04)$. Contudo, quando as variáveis de confundimento foram incluídas, não houve diferença estatisticamente significante.

A avaliação da ingestão alimentar - dieta e suplemento - demonstrou que houve consumo de $107,0 \%$ da necessidade de energia (Figura 2). A ingestão do suplemento oral foi verificada pelo percentual de suplemento consumido sobre o orientado. O consumo de suplemento artesanal foi de Média $(M)=21$, Desvio-Padrão $(D P)=36 \%$ no grupo $B$ e $M=32,5, D P=43 \%$ no grupo $A$, contribuindo com $M=9, D P=15 \%$ no grupo $B$ e $M=21, D P=35 \%$ no grupo $A$ da energia total ingerida. Com relação ao suplemento industrializado, o consumo foi de $M=50,5, D P=39 \%$ no grupo $B$ e $M=64, D P=32 \%$ no grupo $A$. A quantidade média de suplemento consumida contribuiu com a energia total ingerida em $M=26,5, D P=16 \%$ no grupo $B$ e Média - $M=32,5, D P=16 \%$ no grupo A. O volume consumido de $\mathrm{SOI}$ foi maior do que o do SOA, sendo a diferença estatisticamente significante entre o grupo $B(p=0,0018)$ e o grupo $A(p=0,000077)$. A contribuição de energia proveniente dos suplementos também foi maior no SOI, tanto no grupo $B$ quanto no grupo $A$, sendo a diferença significante $(p=0,000012$ e $p=0,00021$, respectivamente). Entretanto, a ingestão energética total e a de proteína de alto valor biológico não apresentaram diferenças signi-ficantes entre os grupos (Figura 2).

Nos pacientes com SOA, foi analisada a influência da dor na ingestão alimentar por meio de análise de associação, incluindo idade, sexo, nível socioeconômico e diagnóstico. Verificou-se associação inversa entre elas: quanto maior a intensidade da dor, menor foi a ingestão alimentar $(r=-7,74 ;$ IC $-15,25$ a $-0,24$ e $p=0,043)$.

Durante o acompanhamento com SOA $27,5 \%(14 / 51)$ dos pacientes do grupo A necessitaram de internação e 39,0\% (21/54) do grupo B. A média de internação foi de 2,1 dias no grupo A e 3,1 dias no grupo B. Dos pacientes que receberam SOI, 33,5\% (8/24) do grupo A e $20,0 \%$ 

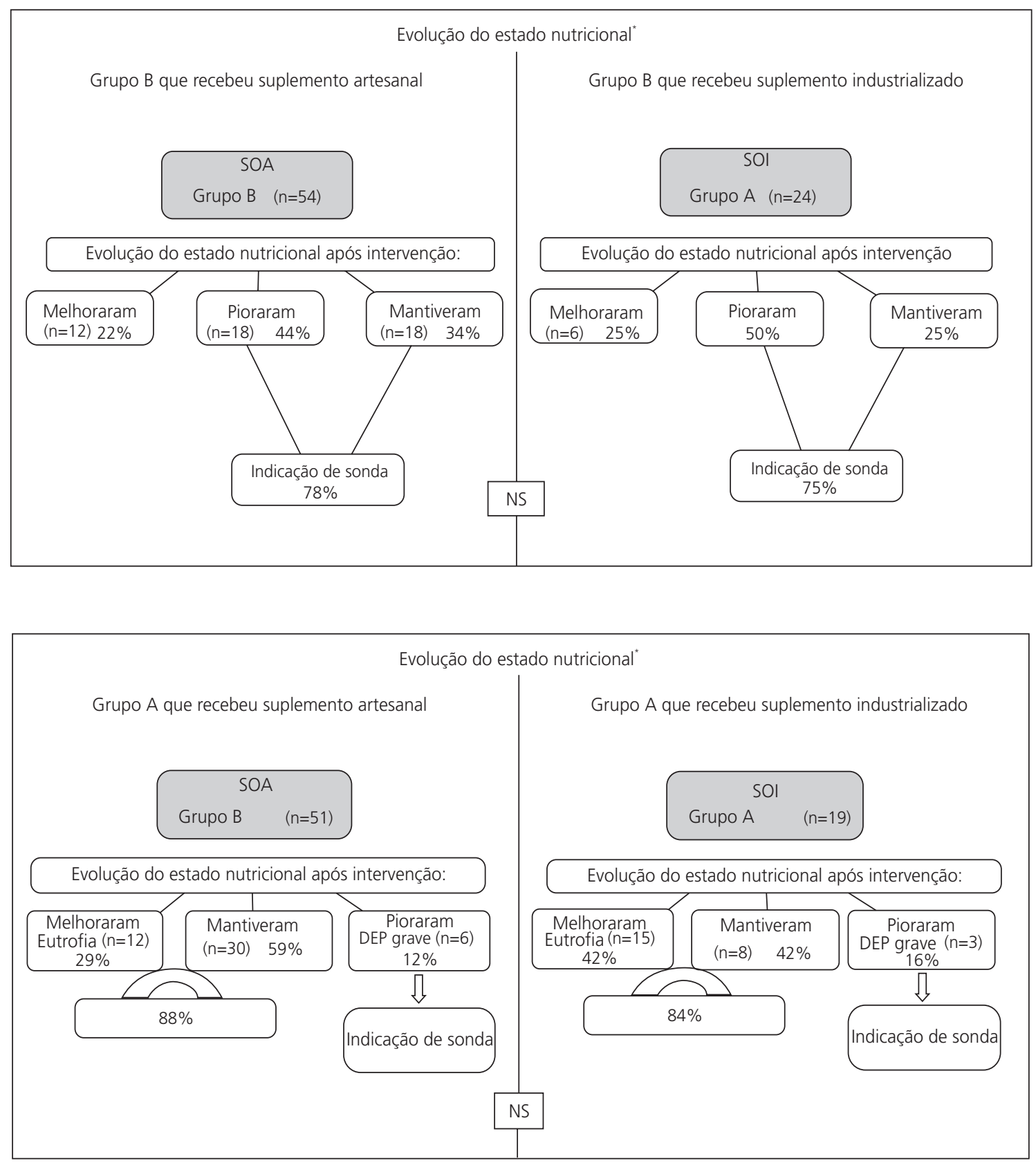

Figura 1. Evolução do estado nutricional nos grupos.

Nota: * O estado nutricional foi avaliado por meio do IMC para adolescentes e escore-Z de peso para estatura para crianças, com os seguintes diagnósticos nutricionais: risco nutricional, desnutrição leve e desnutrição grave.

NS: não significante; SOA: suplementação oral artesanal; SOl: suplementação oral industrializada.

(5/25) do grupo B necessitaram de internação, com média de 2,3 dias de internação no grupo $A$ e 4,6 dias no grupo B. Em ambos os grupos, o principal motivo de internação foi devido às complicações orgânicas ou infecciosas; nos pacientes com SOA, houve $57,0 \%(12 / 21)$ e $64,0 \%(9 / 14)$ no grupo $B$ e $A$, respectivamente. Nos pacientes com SOI, houve $60,0 \%(6 / 5)$ no grupo B e $87,5 \%$ (7/8) no grupo A. Internações por complicações da doença ocorreram somente nos pacientes do 
grupo B com SOA: 19,0\% (4/21). Também houve internações para procedimentos de tratamento, sendo $24,0 \%$ (5/21) no grupo B com SOA, 36,0\% (5/14) no grupo A com SOA; 40,0\% (2/5) no grupo B com SOl e 12,5\% (1/8) no grupo A com SOI.
A capacidade funcional dos pacientes mensurada apresentou resultados significantes no grupo A que recebeu SOI, tanto para os escores de ECOG $(p=0,02)$, quanto para os de Karnofsky ou Lansky $(p=0,04)$. Não houve diferença estatis-

Tabela 2. Evolução do percentual de adequação das pregas e circunferências nos grupos SOI e SOA, separados por estado nutricional.

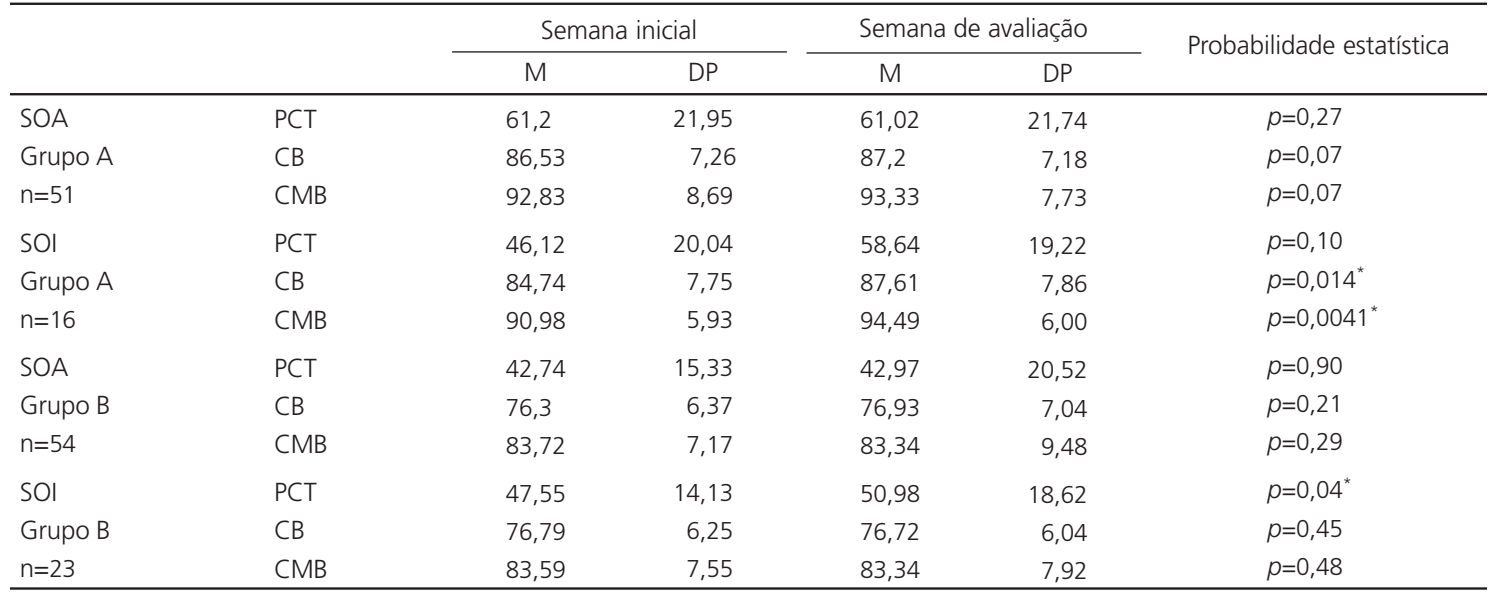

* diferença estatística.

PCT: prega cutânea tricipital; CB: circunferência do braço; CMB: circunferência muscular do braçO; SOA: suplementação oral artesanal; SOI: suplementação oral industrializada; M: média; DP: desvio-padrão.

Grupo A (SOI versus SOA): PCT $(p=0,024), \mathrm{CB}(p=0,011)$ e CMB $(p=0,01)$; Grupo B (SOI versus SOA): PCT $(p=0,16)$.

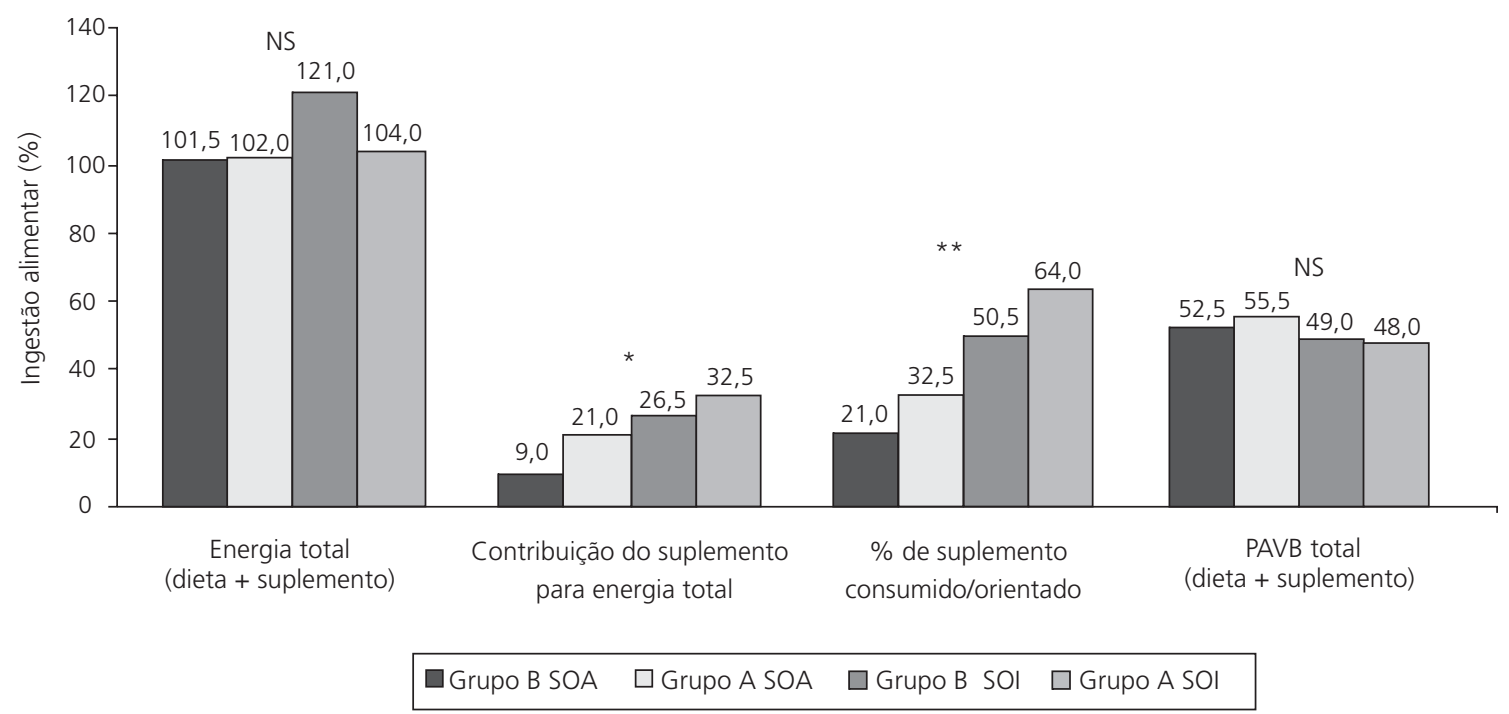

Figura 2. Ingestão alimentar dos pacientes nos grupos SOI e SOA, separados por grupos

Nota: * Grupo B SOA versus SOI ( $p=0,000012)$ e Grupo A SOA versus SOI $(p=0,00021) ;{ }^{* *}$ Grupo B SOA versus SOI $(p=0,0018)$ e grupo A SOA versus SOI ( $p=0,000077) ;$ NS: não significante; PAVB: proteína de alto valor biológico; SOA: suplementação oral artesanal; SOI: suplementação oral industrializada. 
Tabela 3. Evolução do escore de capacidade funcional dos pacientes nos grupos SOI e SOA, separados por grupos.

\begin{tabular}{|c|c|c|c|c|c|c|c|c|}
\hline & & & & \multicolumn{2}{|c|}{ Semana inicial } & \multicolumn{2}{|c|}{ Semana de avaliação } & \multirow{2}{*}{ Probabilidade estatística } \\
\hline & & & & $M$ & $\mathrm{DP}$ & $M$ & DP & \\
\hline Lansky ou & SOA & Grupo A & $n=49$ & 83,06 & 12,62 & 85,10 & 14,45 & $p=0,13$ \\
\hline \multirow[t]{3}{*}{ Karnofsky } & & Grupo B & $\mathrm{n}=44$ & 78,41 & 16,70 & 76,82 & 16,67 & $p=0,76$ \\
\hline & SOI & Grupo A & $n=8$ & 83,75 & 15,06 & 90,00 & 14,14 & $p=0,02^{*}$ \\
\hline & & Grupo B & $n=4$ & 80,00 & 11,55 & 77,50 & 22,17 & $p=0,58$ \\
\hline \multirow[t]{4}{*}{ ECOG } & SOA & Grupo A & $n=49$ & 1,37 & 1,01 & 1,20 & 1,15 & $p=0,16$ \\
\hline & & Grupo B & $n=44$ & 1,75 & 1,08 & 1,73 & 1,04 & $p=0,45$ \\
\hline & SOI & Grupo A & $\mathrm{n}=8$ & 1,13 & 0,99 & 0,75 & 1,04 & $p=0,04^{*}$ \\
\hline & & Grupo B & $n=4$ & 1,5 & 1,29 & 2,25 & 1,5 & $p=0,23$ \\
\hline
\end{tabular}

* diferença estatisticamente significante; SOA: suplementação oral artesanal; SOI: suplementação oral industrializada; M: média; DP: desvio-padrão.

Não houve diferença estatística entre a evolução dos escores de capacidade funcional dos pacientes que receberam SOA e dos que receberam SOI.

ticamente significante entre a evolução dos pacientes que receberam SOI e os que receberam SOA (Tabela 3).

As toxicidades gastrintestinais foram verificadas semanalmente. Foram realizadas 142 avaliações no grupo B que recebeu suplemento artesanal, tendo sido observados 9,0\% (13/142) de mucosite, 20,0\% (28/142) de náusea e vômitos e $6,5 \%(9 / 142)$ de diarreia. No grupo A com suplemento artesanal, foram realizadas 168 avaliações e foram observados 14\% (23/168) de mucosite, 28,5\% (48/168) de náusea e vômito e $10 \%(17 / 168)$ de diarréia. Foram realizadas 68 avaliações no grupo B com suplemento industrializado e observaram-se $19 \%$ (13/68) de mucosite, $31 \%(21 / 68)$ de náusea e vômito e 12\% (8/68) de diarréia. No grupo A também com suplemento industrializado foram realizadas 88 avaliações e observaram-se $8 \%$ (7/88) de mucosite, 18\% (16/88) de náusea e vômito e 4,5\% (4/88) de diarreia. As diferenças percentuais não foram muito expressivas entre os grupos e não houve diferença estatística das toxicidades entre os grupos.

Os pacientes com SOA aderiram mais ao protocolo proposto do que os com SOI $(96,5 \%$ para 87,5\%), embora a diferença não tenha sido significante $(p=0,051)$. Comparando SOI e SOA nos pacientes do grupo $B$, verificou-se que a média de presença não apresentou diferença significante entre esses grupos. A análise de associação entre a indicação de sonda e a frequência nas consultas, incluindo idade, sexo, diagnóstico e escolaridade como variáveis de confundimento, demonstrou relação positiva no grupo SOA: quanto maior a frequência nas consultas, mais indicações de son$d a(r=4,9 ;$ IC 1,25 a 8,85 e $p=0,0089)$.

\section{DIS CUSS Ã O}

A escolaridade pode ser entendida como um indicador indireto do nível social. Quanto menor a escolaridade das famílias, menor é a sua perspectiva econômica ${ }^{20}$. A baixa escolaridade dos cuidadores de ambos os grupos reflete o quão carente é a população deste estudo. Essa realidade justifica a necessidade da terapia nutricional de baixo custo e com composição capaz de recuperar o estado nutricional desses pacientes. Este estudo objetivou testar um suplemento oral aplicável para famílias de baixa renda. Portanto, deve ser ressaltada a importância do atendimento nutricional adequado, investigando se as famílias têm todos os ingredientes e os utensílios para a execução das receitas. Em alguns casos houve a necessidade de adaptar as receitas para algumas realidades específicas, com a preocupação em não alterar a composição idealizada do SOA. Ocorreram, todavia, situações em que alguns ingredientes precisaram ser excluídos ou incluídos, visando melhor aceitação dos pacientes. As modificações das receitas foram realizadas de maneira criteriosa pela equipe de nutrição. Porém, even- 
tualmente, ocorreram alterações realizadas pelos pacientes sem nosso prévio conhecimento. Dos que consumiram o suplemento proposto, 55,0\% não modificaram a receita orientada $(44,0 \%$ grupo B e 66,0\% grupo A). De acordo com o cálculo das dietas, essas alterações não implicaram prejuízos na oferta de macronutrientes. Esses dados são importantes, pois refletem a adesão às receitas dos suplementos desenvolvidos para essa população.

O suplemento artesanal apresenta $11,0 \%$ menos energia quando comparado à preparação do industrializado, representando $80,0 \%$ da energia existente no SOI. Contudo, a comparação dos suplementos mostrou que alguns micronutrientes estão em desvantagem quantitativa no suplemento artesanal. O SOA fornece apenas 9\% da vitamina $C$ ofertada no SOI, $21,0 \%$ da vitamina $\mathrm{K}, 38,0 \%$ da niacina, $49,0 \%$ da vitamina $\mathrm{B}_{6}, 8,0 \%$ do ácido fólico, $15,0 \%$ da biotina, $21,0 \%$ do ferro, $23,0 \%$ magnésio e $51,0 \%$ do zinco. A baixa oferta de vitamina antioxidante e de alguns oligoelementos confere desvantagens ao suplemento proposto. Embora haja diferenças na composição dos suplementos, acreditamos que isso não traga efeitos negativos para a terapia proposta. Apesar das vantagens conhecidas do SOI, é importante ressaltar que o SOA possui maior variabilidade de sabor do que o industrializado em questão, pois mesmo que o SOI seja diluído em leite e que seja adicionado algum ingrediente para dar sabor, o odor e o sabor base do suplemento ainda se mantêm presentes. A proposta de oito receitas objetivou diminuir a monotonia dos suplementos orais, conferindo um efeito positivo para a qualidade de vida desses pacientes e com impacto sobre o estado nutricional próximo ao dos suplementos industrializados.

Os pacientes do grupo SOI consumiram mais suplemento do que os pacientes do grupo SOA, ocorrendo, portanto, maior contribuição de energia no primeiro grupo. Entretanto, não houve impacto direto na energia total ingerida (dieta mais suplemento), bem como no consumo de proteínas de alto valor biológico (Figura 2).
Apesar de globalmente não ter sido verificada diferença significante na ingestão alimentar, a análise de associação demonstrou que a existência da dor refletiu diretamente na ingestão alimentar dos pacientes. A dor é frequente em pacientes com câncer. Segundo Pimenta, a dor crônica acomete cerca de $50 \%$ dos pacientes, e $70,0 \%$ nos estágios mais avançados da doença ${ }^{33}$, e parece intervir diretamente na qualidade de vida desses pacientes ${ }^{34}$. Os resultados deste estudo demonstram que a dor também interfere na ingestão alimentar e, portanto, deve ser um aspecto considerado durante a avaliação e acompanhamento nutricional de todos os pacientes com câncer.

O baixo consumo do SOA pode ter ocorrido devido ao trabalho necessário para a execução da receita. Os pacientes oncológicos possuem uma série de rotinas de tratamento e durante o acompanhamento essa foi a principal dificuldade relatada por alguns cuidadores.

Alguns autores não acreditam que suplementos administrados por via oral possam ser capazes de recuperar o estado nutricional de crianças e adolescentes com câncer. Existem algumas evidências de que os benefícios possam ser maiores nos adultos ${ }^{35,36}$, entretanto um estudo realizado anteriormente a este serviço evidenciou $34,0 \%$ de melhora do estado nutricional de pacientes desnutridos que receberam suplemento oral industrializado 5 . Assim, como nesse serviço, a contribuição dos suplementos orais para o estado nutricional já foi descrita por outros autores e a resposta positiva também foi evidenciada neste estudo ${ }^{4}$. Todavia, os pacientes com maior gravidade nutricional apresentam maior dificuldade para serem recuperados com o emprego de suplementos via oral. Os pacientes do grupo A evoluíram melhor com a terapia oral; observou-se, em média, $86,0 \%$ de resultados positivos. 0 impacto do SOA e do SOI sobre o diagnóstico nutricional desse grupo não foi diferente, conferindo benefícios semelhantes para o fim proposto (Figura 1). 
Um trabalho realizado no serviço verificou que as pregas e as circunferências são bons indicadores do estado nutricional de pacientes oncológicos; por essa razão, também avaliamos tais indicadores ${ }^{5}$. Estes resultados demonstram, de forma geral, que os efeitos benéficos foram mais importantes nos pacientes do grupo A com SOl; no grupo $B$ não foi possível verificar esse mesmo resultado. Como o grupo A foi composto por pacientes com menor gravidade nutricional, a manutenção do estado nutricional pode ser considerada como resultado positivo. Assim, o suplemento nutricional, provavelmente, tenha sido o fator mais relacionado com a melhora dos pacientes do grupo A.

As toxicidades no trato gastrintestinal são efeitos colaterais do tratamento antineoplásico. Mucosite, náusea, vômito e diarreia são alterações que afetam negativamente o estado nutricional dos pacientes, podendo prejudicar a ingestão ali-

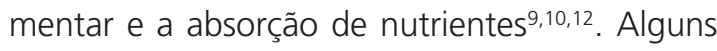
trabalhos sugerem que pacientes desnutridos apresentam mais toxicidades ao tratamento, evoluindo com pior prognóstico ${ }^{3,5}$. Os resultados encontrados foram condizentes com os da literatura. A frequência das toxicidades foi maior nos pacientes do grupo $B$, que apresentavam um pior estado nutricional, tanto no SOI como no SOA, contudo não se observou diferença estatística entre os grupos de estudo.

A adesão à terapia proposta e a frequência às consultas são pontos importantes para a obtenção de resultados positivos. Nem todos os pacientes aderiram ao protocolo proposto e houve menor número de abandonos no grupo com SOA do que no grupo com SOl. Embora os pacientes do grupo com SOA tenham maior número de faltas, a análise estatística não demonstrou diferença significante. Entretanto, para os pacientes do grupo $B$, a probabilidade estatística foi próxima à significância. Acreditamos que a doação do suplemento industrializado pode ter sido o principal fator da maior presença nas consultas. Esse estímulo não existe com o suplemento artesanal; talvez por esse motivo o número de faltas seja maior.
A evolução do escore de capacidade funcional mostrou diferença positiva no grupo A que recebeu SOI. Acreditamos que o período de avaliação foi pequeno para mostrar impacto importante na capacidade de desempenho dos pacientes. Apesar da melhora significante encontrada no grupo A com SOI, a análise entre SOI e SOA não mostrou diferença da evolução dos escores entre os grupos. A média dos escores de Lansky ou Karnofsky atingiu 80,0\%, provavelmente em função da doença de base que resulta em algumas limitações, como cansaço ao esforço e sinais/sintomas específicos da doença. O escore de 80,0\% foi bastante positivo, considerando que a terapia foi proposta para ser realizada em nível ambulatorial. Outro resultado que reforça essa proposta é o número de internações dos pacientes: mais de $50,0 \%$ deles foram acompanhados, exclusivamente, em ambulatório e receberam os suplementos preparados por seus cuidadores.

Os suplementos industrializados apresentam facilidade de preparo, baixo risco de contaminação e adequada composição nutricional. Apesar das vantagens do SOI, existe a desvantagem financeira, principalmente para a aquisição em alguns países, como é o caso do Brasil, onde o SOI custa cinco vezes mais do que o SOA.

A terapia nutricional com SOA demonstrou desempenho que a coloca dentre as opções para pacientes oncológicos em tratamento; acreditamos, assim, que o emprego da terapia com SOA possa auxiliar a recuperação nutricional desses pacientes, sendo uma opção para populações financeiramente desfavorecidas. A variabilidade de sabores associada ao baixo custo faz dele um suplemento que também poderá ser utilizado por outras populações.

\section{COLABORADORES}

F.R. ALVES participou do desenvolvimento das receitas, da coleta e da análise dos dados, da discussão, dos resultados e da elaboração do artigo. A. GARÓFOLO participou da idealização do projeto com desenvolvimento das receitas, análise dos dados, discussão 
dos resultados e finalização do manuscrito. P.S. MAIA participou do desenvolvimento das receitas e da coleta dos dados. F.J. NÓBREGA participou da discussão dos resultados e da finalização do manuscrito. A.S. PETRILLI participou da discussão dos resultados e da finalização do manuscrito.

\section{REFERÊ NCIAS}

1. Instituto Nacional do Câncer [Internet]. [acesso 2007 mar. 8]. Disponível em: <http://www.inca. gov.br>.

2. National Cancer Institute. Surveillance epidemiology and end results. [cited 2007 Aug. 5]. Available form: $<$ http://seer.cancer.gov/>.

3. Jain V, Dubey AP, Gupta SK. Nutrition parameters in children with malignancy. Indian Pediatr. 2003; 40(10):976-84.

4. Garófolo A, Lopez FA, Petrilli AS. Acompanhamento do estado nutricional de pacientes com osteossarcoma. Acta Oncol Bras. 2002; 22(1):233-7.

5. Garófolo A, Loez FA, Petrilli AS. High prevalence of malnutrition among pacients with solid non-hematological tumors as found by using skinfold and circunference measurements. São Paulo Med J. 2005; 123(6):277-81.

6. Garófolo A, Caran EM, Silva NS, Lopez FA. Prevalência de desnutrição em crianças com tumores sólidos. Rev Nutr. 2005; 18(2):193-200. doi: 10.1590/S14 15-52732005000200003.

7. von Meyenfeldt M. Cancer-associated malnutrition: an introduction. Eur J Oncol Nur. 2005; 9(1002): S35-38.

8. Fluentes M, Sanchez C, Granados MA, Boscan A, Rojas N. Evaluación del estado nutricional en niños con cáncer. Rev Venez Oncol. 2007; 19(3):204-9.

9. Garófolo A, Petrilli AS. Balanço entre ácido graxo omega-3 e 6 na resposta inflamatória em pacientes com câncer e caquexia. Rev Nutr. 2006; 19(5):611-21. doi: 10.1590/S1415-52732006000500009.

10. Ravasco P. Aspects of taste and compliance in patients with cancer. Eur J Onc Nurs. 2005; 9:S84-91.

11. Luisi FAV, Petrilli AS, Tanaka C, Caran EMM. Contribution to the treatment of nausea and emesis induced by chemotherapy in children and adolescents with osteossarcoma. São Paulo Med J. 2006; 124(2):61-5.

12. Garófolo A, Boin SG, Modesto PC, Petrilli AS. A avaliação da eficiência da nutrição parenteral quanto à oferta de energia em pacientes oncológicos pediátricos. Rev Nutr. 2007; 20(2):181-90. doi: 10.1590/S1415-52732007000200007.
13. Jackson AA, Ashworth A, Khanum S. Improving child survival: malnutrition task force and the paediatrician's responsibility. Arch Dis Child. 2006; 91(8):706-10.

14. Maitland K, Berkley JA, Shebbe M, Peshu N, English $M$. Children with severe malnutrition: can those at highest risk of death be identified with the WHO protocol? PLoS Med. 2006; 3(12):2431-9.

15. Sarni ROS, Souza FIS, Catherino P, Kochi C, Oliveira FLC, Nobrega FJ. Tratamento de criança com desnutrição grave utilizando o protocolo da OMS: experiência de um centro de referência, São Paulo/Brasil. Arch Latinoam Nutr. 2005; 55(4):336-44.

16. Deen JL, Funk M, Guevara VC, Saloojee H, Doe JY, Palmer A, et al. Implementation of WHO guidelines on mamagement of severe malnutrition in hospitals in Africa. Bull World Health Organ. 2003; 81(4): 237-43.

17. Sarni RO, Souza FIS, Catherino P, Kochi C, Oliveira FLC, Nobrega FJ. Tratamento da desnutrição em crianças hospitalizadas em São Paulo. Rev Assoc Med Bras. 2005; 51(2):106-12.

18. Sawaya AL. Desnutrição: consequências em longo prazo e efeitos da recuperação nutricional. Estud Av. 2006; 20(58):147-58.

19. Odelli C, Burgess D, Bateman L, Hughes A, Ackland S, Gilliest J, et al. Nutrition support improves patient outcomes, treatment tolerance and admission characteristics in oesophageal cancer. Clin Oncol. 2005; 17(8):639-45.

20. FGVDADOS Fundação Getúlio Vargas [Internet]. [acesso 2007 maio 5]. Disponível em: <http://www. fgvdados.fgv.br>

21. Institute of Medicine. Dietary reference intakes: applications in dietary assessment. Washington (DC): National Academy Press; 2000.

22. National Research Council. Recommended dietary allowances. $10^{\text {th }}$ ed. Washington (DC): National Academy Press; 1989.

23. Children's Oncology Group Cancer Control. Nutrition Sub-Committee. Algorithm for nutrition intervention and categories of nutritional status in the pediatric oncology patient-references and resources. Washington (DC); 2004.

24. Sacks N, Meek RS. Nutritional support. In: Arthur RA. Supportive care of children with cancer. Current therapy and guidelines from the children's Cancer Group. $2^{\text {nd }}$ ed. Baltimore: The Johns Hopkins University Press; 1997 . p.193.

25. Lansky SB, List MA, Lansky LL, Ritter-Sterr C, Miller DR. The measurement of performance in childhood cancer patients. Câncer. 1987; 60(7):1651-6. 
26. Karnofsky DA, Burchenal JH. The clinical evaluation of chemotherapeutic agents in cancer. In: MacLeod CM (Ed). Evaluation of Chemotherapeutic Agents. New York: Columbia Univ Press; 1949.

27. Oken MM, Creech RH, Tormey DC, Horton J, Davis TE, McFadden ET, et al. Dairs TE. Toxicity and response criteria of the Eastern Cooperative Oncology Group. Am J Clin Oncol. 1982; 5(6):649-55.

28. Thompson FE, Byers T. Dietary assessment resource manual. J Nutri Suppl. 1994; 124(11 Suppl): S2245-317.

29. McGrath PJ. Acute pains. In. McGrath PJ, editor. Pain in children. New York: Guilford Press; 1990. 208-50.

30. Claro MT. Escala de faces para avaliação da dor em crianças: etapa preliminar [tese]. Ribeirão Preto: Universidade de São Paulo; 1993.

31. Beyer JÊ. Knapp TR. Methodological issues in the measurement of children'pain. Child Health Care. 1986; 14(4):233-41.
32. Dawson B, Trapp RG. NCSS [computer program]. Version NCSS/PASS 2000: statistical software Dawson edition. Rio de Janeiro: McGraw-Hill; 2003.

33. Pimenta CAM, Koizumi MS, Teixeira MJ. Dor no doente com câncer: características e controle. Rev Bras Cancerol. 1997; 43(1):21-44.

34. Silva LMH, Zago MMF. O cuidado do paciente oncológico com dor crônica na ótica do enfermeiro. Rev Latino-Am Enfermagem. 2001; 9(4):1-8.

35. Pencharz PB. Aggressive oral, enteral or parenteral nutrition: prescriptive decisions in children with cancer. Int J Cancer. 1998; 78(S11):73S-75S.

36. Richard JA, Chwals WJ. Nutritional support of the pediatric oncology patient. Nutrition. 1998; 14(1): 124-9

Recebido em: 9/1/2009

Versão final reapresentada em: 3/12/2009 Aprovado em: 11/5/2010 